\title{
Impact of lung surfactant on wettability and cytotoxicity of nanoparticles
}

Cite this: RSC Adv., 2014, 4, 20573

\begin{abstract}
Monica Ratoi, ${ }^{\star a}$ Peter H. M. Hoet, ${ }^{\text {b }}$ Alison Crossley ${ }^{c}$ and Peter Dobson ${ }^{d}$
The importance of nanoparticle characterization for nanotoxicology has been extensively emphasized and it has been universally agreed that the most important parameters for characterizing nanomaterials are specific surface area and surface properties (chemistry, hydro-philicity/phobicity, charge etc.). This study is proposing the use of enthalpy of wetting which depends on both specific surface area and surface properties, is easily measurable and proves to be highly relevant for predicting nanoparticles' dispersion state and their interaction with the lungs. It also shows the conditioning effect of the lung surfactant main component, DPPC on the surface of particles when used in concentrations which mimic pulmonary exposure more closely.
\end{abstract}

Received 6th December 2013 Accepted 14th April 2014

DOI: $10.1039 / \mathrm{c} 3 \mathrm{ra} 47374 \mathrm{k}$

www.rsc.org/advances alveoli collapse by reducing the lung air interfacial tension. ${ }^{12}$ Dipalmitoylphosphatidylcholine (DPPC) together with proteins SP-B and SP-C are the main contributors to the surface activity of the pulmonary surfactan $t^{13}$ and a large number of studies used DPPC as a surrogate of lung surfactant. ${ }^{1,4-6,14-24}$

Through their adsorption on solid surfaces, surfactants can modify their wettability. ${ }^{25,26}$ Wettability of NPs is particularly important for understanding nanoparticle aggregation, dissolution and bioaccumulation. ${ }^{27}$ Surface wettability, the degree to which a fluid spreads on a solid surface, depends on the nature of the surface and the fluid involved. Used in concentrations higher than the critical micelle concentration, surfactants adsorb as monolayers on hydrophobic surfaces/particles (i.e. carbon, polymers, etc.) and form bilayers on hydrophilic ones (i.e. metal, metal oxides, glass, ceramics). ${ }^{28-30}$ This is also the case of the zwitterionic surface agent DPPC which in high concentrations, adsorbs on hydrophilic surfaces as multiple bilayers $(5-6 \mathrm{~nm}$ in thickness) which trap a $2 \mathrm{~nm}$ layer of water in between them. ${ }^{31}$ The complete DPPC bilayers expose the hydrophilic moieties and provide polar, water 'wettable' surfaces.

The lamellar liquid crystals formed by the lung surfactant in hypophase contain abundant amounts of DPPC and enables adsorption of complete DPPC bilayers on hydrophilic particles and monolayers on hydrophobic particles. Regardless of the wettability of particles, through its adsorption on their surface, DPPC renders them hydrophilic (Fig. 1) and consequently influences their dispersibility and surface charge.

Numerous studies investigated the dispersion and toxicity of fine and ultrafine particles in DPPC or BAL (brochoalveolar lavage) aqueous media. The concentration of the DPPC/BAL varied widely and generated potentially conflicting outcomes of both, dispersion state and toxicity of particles as it has been emphasized by several authors. ${ }^{1,4,7,32}$ Wallace and et al..$^{6,14-22}$ investigated and revealed the importance of employing high
${ }^{a}$ Faculty of Engineering and Environment, University of Southampton, Highfield Campus, Southampton SO17 1BJ, UK. E-mail: m.ratoi@soton.ac.uk

${ }^{b}$ Laboratory of Pneumology, Unit for Lung Toxicity, K. U. Leuven, Herestraat 49, 3000 Leuven, Belgium

'Department of Materials, University of Oxford, UK

${ }^{d}$ Begbroke Science Park Directorate, University of Oxford, UK OX5 1PF 

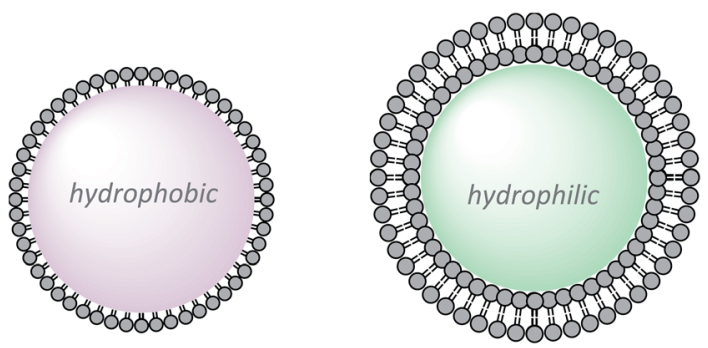

Fig. 1 Schematics of phospholipid adsorption as a monolayer on hydrophobic surface NPs (i.e. polymers, etc.) and as bilayers on hydrophilic surface NPs (i.e. metals, metalloids, oxides, etc.). The phospholipid coating renders the surface of all NPs hydrophilic (water wettable) when used in concentrations which mimic in vivo conditions.

DPPC concentrations in particle toxicity studies. Other authors ${ }^{5,23,33}$ who followed Wallace's recommendation reported similar toxicology results. Despite the evidence of these reported findings, the majority of published research used diluted DPPC/BAL dispersion media which did not promote a complete phospholipid mono (on hydrophobic NPs) or bilayer (hydrophilic surface) coverage. An incomplete DPPC monolayer/ bilayer coverage alters the wettability of the particles and it can convert hydrophilic particles into hydrophobic. This explains the outcomes of many studies which reported that DPPC/BAL dispersion media led to particle agglomeration, sedimentation and various contradictory toxicity effects.

Wetting is primarily an enthalpy driven process and the enthalpy of wetting is directly related to parameters which have been linked to toxicity of NPs such as: surface area, surface charge, chemistry and solubility and has the potential to be a valuable characterization parameter. ${ }^{34,35}$

This study has investigated how DPPC adsorbs on the titania P25 NPs and influences their dispersion and cytotoxicity. It also introduces a novel characterization parameter, the enthalpy of wetting of NPs and explores its potential to predict the NPs dispersion and interaction with biological systems.

\section{Materials and methods}

\subsection{Test particles}

Aeroxide P25 titania NPs were supplied by Evonik Industries AG. The primary particle size, specific surface area and tamped density supplied by the manufacturer in the product information sheet, are listed in Table 1. The phase composition also shown in Table 1 was calculated from XRD analysis. The $\mathrm{pH}$ of point of zero charge (PZC) of titania P25 NPs measured in deionized water (DI) was 6.7.

\subsection{DPPC coating of $\mathbf{P 2 5}$ titania nanoparticles}

1,2-Dipalmitoyl-sn-glycero-3-phoshocholine (DPPC) $\geq 99 \%$ ( $M=$ 734.04) was purchased from Sigma Aldrich, UK.

The coating of the NPs with DPPC followed the procedure proposed by Wallace and collaborators. ${ }^{6}$ The surfactant dispersion was prepared at $37^{\circ} \mathrm{C}$ by ultrasonically mixing DPPC in physiological saline (PSS) at $1.25 \mathrm{mg}$ DPPC/1 ml PSS. Although surface active, lecithin has very low solubility in water. Titania P25 NPs were ultrasonically mixed into the surfactant dispersions in $1 \mathrm{mg}$ NPs $/ 1.25 \mathrm{mg}$ DPPC/ $1 \mathrm{ml}$ PSS and gently stirred in an incubator at $37{ }^{\circ} \mathrm{C}$ for one hour. NPs were centrifuged (4025 G-force) and the supernatant decanted. Additional DI water was added to the pellet and the centrifuge-washing step repeated three times. The DPPC coated P25 NPs were freeze-dried to a powder. This procedure ensured that the titania NPs were fully covered in lipid but only a single bilayer remained as showed by the Raman analysis and wettability results.

To investigate the dispersibility of the pristine and DPPC coated NPs in DI water (Millipore Nanopure Water), the dispersions were prepared in three concentrations: $C_{1}=1000$ $\mu \mathrm{g} \mathrm{ml} l^{-1}, C_{2}=100 \mu \mathrm{g} \mathrm{ml}^{-1}$ and $C_{3}=10 \mu \mathrm{g} \mathrm{ml}^{-1}$. NP dispersions in $C_{1}$ were sonicated for 15 minutes using a bath sonicator before dilution to $C_{2}$ and $C_{3}$. Vortexing at maximum power for 15 seconds was employed before dilutions and the measurement of particle size distribution and surface charge. The $\mathrm{pH}$ of the NP dispersions in DI water was approximately 7.

\subsection{Characterization of NP dry powders}

2.3.1. BET specific surface area. Specific surface area measurements have been carried out on a Micromeritics Gemini V BET surface area analyser. Prior to measurements, samples of 150-200 mg have been degassed in nitrogen atmosphere overnight at $25{ }^{\circ} \mathrm{C}$. Two measurements were carried out for each sample.

2.3.2. Raman spectroscopy. Raman spectroscopy analysis was performed using a microRaman spectrograph (Horiba LabRAM Aramis integrated confocal system $460 \mathrm{~mm}$ focal length) with an internal He-Ne laser $633 \mathrm{~nm}$ and $20 \mathrm{~mW}$ output power. The laser source was focused on the samples through a long working distance $50 \times$ objective to a spot diameter of $2 \mu \mathrm{m}$. The acquisition time for Raman spectra was 10-20 minutes depending on the strength of the Raman signals, until a satisfactory signal-to-noise ratio was achieved.

2.3.3. Microcalorimetry. The heat of wetting/dewetting of NPs was measured with the TA Instruments Relative Humidity (RH) perfusion microcalorimeter equipped with the TAM III thermostat. The microcalorimeter is a heat-flow calorimeter of the twin-type which uses a $20 \mathrm{ml}$ ampoule. It was preferred to

Table 1 P25 titania NP parameters

Mean primary particle size diameter $(\mathrm{nm})$
BET specific surface area $\left(\mathrm{m}^{2} \mathrm{~g}^{-1}\right)$
Tamped density $\left(\mathrm{g}^{-1}\right)$
Composition (wt $\%)$ 
the nanocalorimeter because the ampoule size permits a larger contact area of the water/solvent vapours with the nanopowder and can therefore reveal smaller differences in the wettability of samples.

The RH was kept constant at $10 \%$ until a stable heat flow was reached (signal within the range of -1 to $+1 \mu \mathrm{W}$ ). The $\mathrm{RH}$ was then increased in a linear ramp from $10 \%$ to $90 \%$ over the following 10 hours and maintained at $90 \%$ for 10 hours. During the increase of humidity, the moisture interacts with the sample and produces a heat flow, called heat of wetting. The TAM III was housed in a thermostatic room maintained at $25 \pm 1{ }^{\circ} \mathrm{C}$ and it was calibrated with empty stainless steel ampoules before the experiments. The measurements were carried out on 35-50 mg of samples and were repeated twice.

\subsection{Characterization of NP dispersions}

2.4.1. Particle size distribution and charge. The hydrodynamic size distributions of the NP dispersions were measured with the Malvern Zetasizer and CPS Disc Centrifuge (CPS Disc Centrifuge Model DC24000, CPS Instruments, Europe) which are Dynamic Light Scattering (DLS) techniques. In the CPS Centrifuge, dispersion samples of $300 \mu \mathrm{l}$ were analyzed at $12000 \mathrm{RPM}$. The ZetaSizer Nano ZS (Malvern Instruments, UK) equipped with MPT-2 Multipurpose Titrator was employed for measurements of hydrodynamic particle size distribution, surface charge (zeta potential) and point of zero charge (PZC).

The titania dispersions were prepared in DI water rather than other biological media to be able to compare the particle size and surface charge results with the wettability values (heat of wetting/dewetting of water vapours). Prior to the size and zeta potential measurements, titania dispersions were sonicated for 15 minutes using a bath sonicator and vortexed for 15 seconds before each dilution. All measurements were carried out at $25{ }^{\circ} \mathrm{C}$. Repeatability of all hydrodynamic size and zeta potential was checked more than three times.

\subsection{Cell culture}

A549 cells from a human lung adenocarcinoma with alveolar type II phenotype were obtained from Salisbury, UK (ECACC \#8601284). Cells were cultured in RPMI 1640 with $25 \mathrm{mM}$ HEPES and Glutamax (Gibco \#72400-021), 10\% FBS (ATCC \#302021), 1\% penicillin-streptomycin (100× Gibco \#15140-122), $0.5 \%$ fungizone $(100 \times$ Gibco \#15290-018) and tryspin-EDTA (10× Gibco \#15400-054) in a humidified atmosphere at $37^{\circ} \mathrm{C}$.

A cytotoxicity dose-response curve was created for the different nanoparticles, in the presence or absence of DPPC, using the thiazolyl blue tetrazolium bromide (MTT) or lactate dehydrogenase (LDH) assay. The cells were seeded at a density of $100 \times 10^{3}$ cells per $\mathrm{cm}^{3}$ and medium was removed 24 hours later. The cultures were exposed to 3.3, 10, 33, 100, 333, 1000, $3333 \mu \mathrm{g}$ nanoparticles per ml. DPPC alone was dosed at a 10 fold lower concentration. ${ }^{36}$ In these experiments, controls and negative controls were included as described in. ${ }^{36,37}$

2.5.1. MTT assay. After $24 \mathrm{~h}$ of exposure, the medium was removed and cells were rinsed with HBSS. The MTT solution (0.5 $\mathrm{mg} \mathrm{ml}^{-1}$ MTT in HBSS) was added for 3 hours. Thereafter, the MTT solution was removed, and $100 \mu \mathrm{l}$ of DMSO was added. The optical density was read at $550 \mathrm{~nm}$ with $655 \mathrm{~nm}$ as reference wavelength, using a microplate reader (Bio-Rad). Cells not incubated with nanoparticles and/or DPPC served as 100\% viability control ( $n=6$ wells in 2 separate experiments).

2.5.2. LDH assay. Cells integrity was assessed by determining the percentage of $\mathrm{LDH}$ retained by the cellular layer. For LDH assay, after incubation with particles, the medium was removed; the adherent cells were washed twice with PBS $(200 \mathrm{ml})$ and then lysed using Triton-X $(0.2 \%)$. The LDH activity of the medium (LDH medium) and the cell lysate (LDH cells) were determined spectrophotometrically monitoring the reduction of pyruvate. Cell viability was calculated according to the formula:

$$
\begin{aligned}
\% \text { viability }= & {[\mathrm{LDH} \text { cells } /(\mathrm{LDH} \text { cells }+\mathrm{LDH} \text { medium })] } \\
& \times 100(n=6 \text { wells in } 2 \text { separate experiments }) .
\end{aligned}
$$

\section{Results and discussion}

\subsection{Adsorption of lung surfactant on NPs}

The surface of P25 titania NPs dispersed in water is generally covered by hydroxyl groups. ${ }^{38}$ Having a polar surface, titania NPs are hydrophilic and will adsorb at least a bilayer of DPPC on their surface. In the current study, the calculated amount of DPPC necessary to cover with a complete bilayer $1 \mathrm{mg}$ P25 titania NPs was $0.32 \mathrm{mg}$ (considering the surface area per DPPC molecule of $40 \AA^{2}$ and the specific surface area of P25 NPs of $52.58 \mathrm{~m}^{2} \mathrm{~g}^{-1}$ ). To ensure the DPPC concentration was sufficiently high to form a complete bilayer on NPs, we started with the amount ratio recommended by Wallace ${ }^{6}$ of $2.50 \mathrm{mg}$ DPPC/1 $\mathrm{ml}$ PSS/1 mg NPs.

This DPPC amount was difficult to disperse in PSS and it was diluted down to $1.25 \mathrm{mg}$ DPPC/1 ml PSS. $1 \mathrm{mg}$ NPs was mixed in the phospholipid dispersion which contained four times the DPPC amount necessary for complete bilayer coverage. The excess DPPC was removed by multiple rinsing with DI water.

The recommendations of Wallace and et al. for the DPPC/ NPs wt ratio were based on data from unpublished investigations. In earlier published studies of microsized silica particles, Wallace employed the wet phosphate assay to quantify the particle uptake of DPPC from PSS dispersions at $37{ }^{\circ} \mathrm{C}$ over a range of DPPC dispersion concentrations. Two types of silicate particles, quartz and kaolin with specific surface area values of 4 $\mathrm{m}^{2} \mathrm{~g}^{-1}$ and $13 \mathrm{~m}^{2} \mathrm{~g}^{-1}$ respectively were found to adsorb 50-60 $\mathrm{mg}$ and $150 \mathrm{mg}$ lecithin per $\mathrm{g}$ as multilayers. ${ }^{15,19}$

The $2.50 \mathrm{mg}$ DPPC/1 $\mathrm{mg}$ NPs wt. ratio recommended by Wallace $^{6}$ is indeed high but it is the amount required to assure a complete bilayer coverage of NPs such as polar (i.e. surface functionalized) CNTs because of their very high specific surface area (i.e. $2.2-3 \mathrm{mg}$ DPPC are necessary to achieve complete bilayer coverage of $1 \mathrm{mg}$ CNT with $S_{\mathrm{SA}}=490 \mathrm{~m}^{2} \mathrm{~g}^{-1}$ ). The excess DPPC can be rinsed off from the coated NPs because the complete monolayer/bilayer is stable to water rinsing. ${ }^{6,39}$

The presence of the DPPC bilayer coating on NPs was tested with Raman spectroscopy. The Raman spectra for the DPPC coated P25 NPs, P25 NPs and DPPC powder are shown in Fig. 2. 


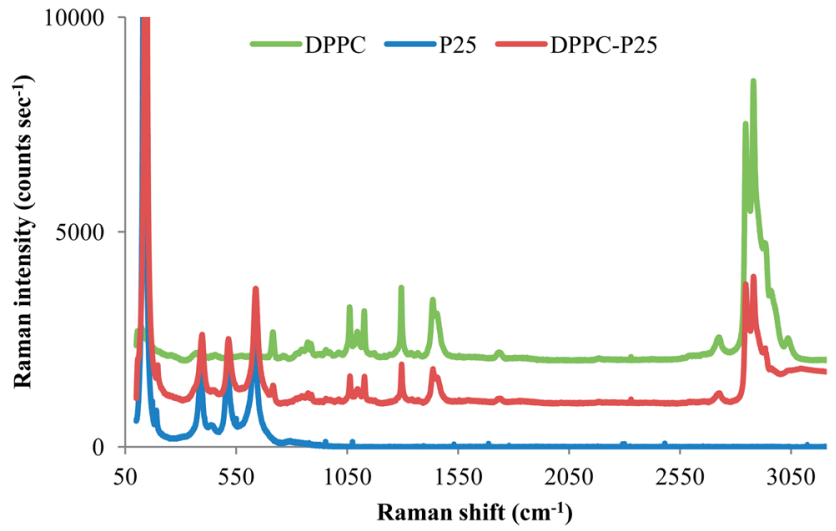

Fig. 2 Raman spectra for P25 NPs, DPPC coated P25 NPs and DPPC powder.

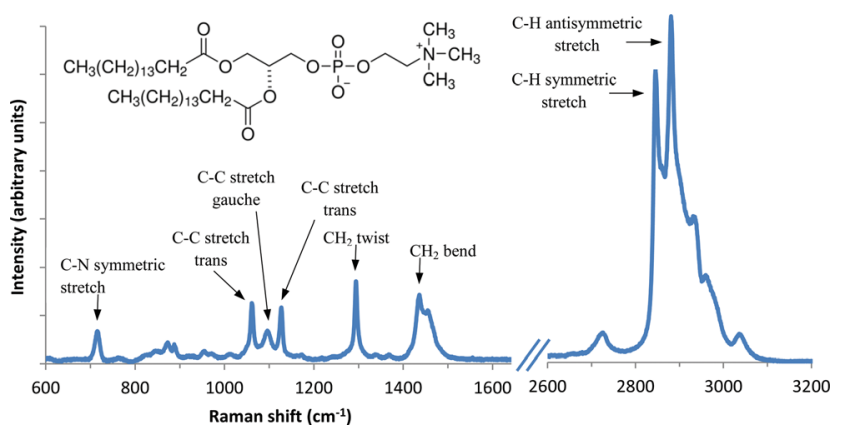

Fig. 3 Raman spectrum of DPPC powder, showing structure of DPPC and band assignments.

For DPPC powder, a representative spectrum similar to other published spectra ${ }^{40}$ is shown in Fig. 3 with band assignments. Spectral peaks can be found in the fingerprint region (600$\left.1800 \mathrm{~cm}^{-1}\right)$ associated with choline symmetric stretch $\left(718 \mathrm{~cm}^{-1}\right)$ and the lipid hydrocarbon acyl chains: $(1060,1100$, and $1128 \mathrm{~cm}^{-1}$ ) but the most intense bands in the spectrum are the $\mathrm{C}-\mathrm{H}$ stretching region $\left(2800-3100 \mathrm{~cm}^{-1}\right)$. The methylene vibrations at $2845,2880,2930$ are sensitive to conformational changes as well as intermolecular interactions of the alkyl chains of lipids and therefore has been much research focused on their interpretation. The Raman spectra for DPPC-P25 NP as compared to DPPC powder show a change in intensity ratio for the $2850 / 2880 \mathrm{~cm}^{-1}$ bands. The intensity ratio, $I_{2880} / I_{2845}$ derived from these spectra, serves as an index of the strength of the lateral interchain packing interactions in the bilayer. ${ }^{41}$ The intensity ratio value for DPPC-P25 is 1.04 similar to that found by other researchers for supported DPPC bilayers on $\mathrm{SiO}_{2} \mathrm{NPs}$ with a diameter of $30-40 \mathrm{~nm} .{ }^{42}$

\subsection{Effect of DPPC coating on wettability, dispersibility and surface charge of NPs}

For solid surfaces, the most popular parameter used to describe wettability is the contact angle. For powders, two main methods have been traditionally used for determining the contact angle: measurements on a compacted powder surface and of the penetration into loosely packed beds but because of the irregular geometry of the systems both present problems. ${ }^{43,44}$ The penetration experiment uses the Washburn equation which has been shown to be flawed ${ }^{45}$ and in the case of compressed powder, the effect of compression affects the values of the contact angle. A newer, simple approach was proposed by Shanker, ${ }^{46}$ in which the powder is glued to an inert support but this method also has its practical drawbacks. Recently, two novel techniques for determination of contact angles of NPs were proposed: multi-angle single-wavelength ellipsometry ${ }^{47}$ and freeze-fracture shadow-casting cryo-scanning electromicroscopy ${ }^{48}$ These methods also have shortcomings and are yet at the experimental stage.

Isothermal microcalorimetry, which measures the heat changes associated with the wetting process, is a sensitive and straightforward way to measure wettability of powders. Measurements are carried under controlled environmental conditions and can also be carried out for hydrophobic powders. Generally, the heats of immersion in water are high for the hydrophilic surfaces (indicating a strong interaction with water molecules) and low for the hydrophobic ones. ${ }^{49}$

Enthalpy of wetting is the heat of wetting (measured with isothermal calorimetry) divided by the specific surface area of the powder and depends on the nature of the surface (chemistry and roughness) and the dissolution (solubility) in the wetting fluid. For a number of inorganic oxides, a linear relation has been found to exist between the heat of wetting and the point of zero charge (PZC). ${ }^{34,35}$

Fig. 4 shows the thermograms for wetting and de-wetting by water of the surface of P25 and DPPC coated P25 NPs. The humidity has been increased and decreased from 10 to $90 \%$.

Table 2 shows the measured values of the heat of wetting/dewetting with water and the BET specific surface area of the P25 and DPPC coated P25. All measurements were carried out at $25{ }^{\circ} \mathrm{C}$ and were repeated at least once.

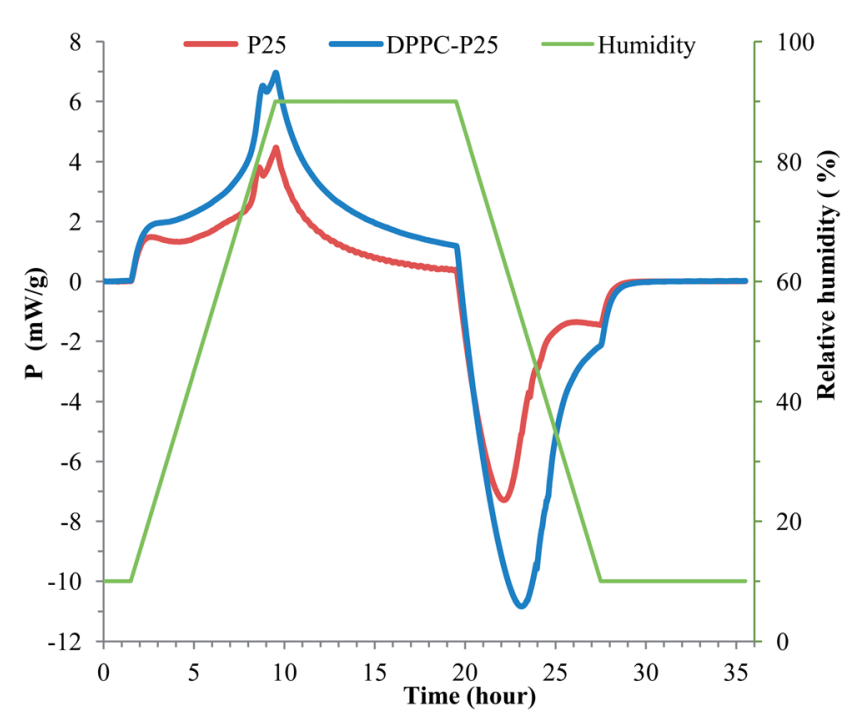

Fig. 4 Thermograms for wetting/dewetting of P25 and DPPC coated P25 NPs. 
Table 2 Surface area, heat and enthalpy of wetting of P25 and DPPC coated P25 NPs

\begin{tabular}{lll}
\hline & P25 & DPPC-P25 \\
\hline $\begin{array}{l}\text { BET specific surface } \\
\text { area }\left(\mathrm{m}^{2} \mathrm{~g}^{-1}\right)\end{array}$ & $52.58 \pm 3$ & $14.54 \pm 1$ \\
$\begin{array}{l}\text { Heat of wetting/dewetting } \\
-\Delta h_{\mathrm{w}} / \Delta h_{\mathrm{dw}}\left(\mathrm{mJ} \mathrm{g}^{-1}\right)\end{array}$ & $91.8 / 91.99 \pm 2$ & $179.5 / 173.6 \pm 1$ \\
$\begin{array}{l}\text { Enthalpy of wetting } \\
-\Delta H_{\mathrm{w}}\left(\mathrm{mJ} \mathrm{m}^{-2}\right)\end{array}$ & 1.7 & 12.3
\end{tabular}

Both samples show an exothermic heat flow (negative heat of wetting) during moisture adsorption (increasing humidity) and endothermic heat flow during moisture desorption which indicates that the surfaces of NPs are hydrophilic. The heat of immersion enthalpy (absolute value) and the surface free energy increase with the hydrophilicity of the sample. By integrating from a $10 \% \mathrm{RH}$ equilibrium through to $90 \% \mathrm{RH}$ and reversing to $10 \% \mathrm{RH}$ equilibrium there is a significant difference in the moisture interaction (wettability) between samples. The DPPCNPs have larger negative values of heat than the non-coated NPs which indicates that the DPPC coating increased the hydrophilicity of P25 NPs.

Published work which measured the surface free energy and its components to study wettability of spin coated DPPC applied on hydrophilic materials (slides of glass, silica and mica) reported similar findings. The concentration of the DPPC was found to exert a major influence on the wettability of the coating. The hydrophilic character of the coating decreased when DPPC concentrations were up to $0.5 \mathrm{mg} \mathrm{ml} \mathrm{m}^{-1}$ and it increased and levelled off when $2-2.5 \mathrm{mg} \mathrm{ml}^{-1}$ was used. The advancing contact angle of water in the second case was $10 \pm$ $2.6^{\circ}$ which means that water almost completely spreads on the DPPC coating. ${ }^{50}$

The enthalpy of wetting is calculated by dividing the measured heat of wetting by the BET specific surface area.

The measured BET specific surface areas for the pristine and DPPC coated P25 NPs are shown in Table 2. It is known that the surface area and porosity of NPs can change as a result of adsorption of biomolecules or agglomeration. ${ }^{27}$ Our results show that the surface area of DPPC-P25 NPs has been substantially decreased by the DPPC bilayer adsorption on P25 NPs. BET surface area measurements were also carried out on pristine and DPPC coated rutile titania NPs (our unpublished data) and a similar degree of reduction in specific surface area was recorded. The particle size distribution data which will be presented later showed that the agglomeration of the NPs did not contribute to the size decrease because the particle size distributions of DPPC-P25 dispersion in DI water indicate much lower average sizes and polydispersity than the pristine P25 NPs. Therefore, we considered that this decrease in specific surface area can be due to two main factors. First, in the calculation of the specific areas of both, the pristine and DPPC coated P25 NPs, the same tapped density value of $1.3 \mathrm{~g} \mathrm{~cm}^{-3}$ (supplied by the manufacturer) was used because the tapped density value for DPPC-P25 NPs wasn't available. However, the
Table 3 Surface charge of NP dispersions in DI water in three concentrations

\begin{tabular}{lll}
\hline & $\begin{array}{l}\text { Concentration } \\
\left(\mu \mathrm{g} \mathrm{ml} \mathrm{ml}^{-1}\right)\end{array}$ & $\begin{array}{l}\text { Av. zeta potential } \\
\text { at } 22{ }^{\circ} \mathrm{C}(\mathrm{mV})\end{array}$ \\
\hline P25 & $C_{1}=1000$ & -0.5 \\
& $C_{2}=100$ & -21.1 \\
& $C_{3}=10$ & -24.2 \\
DPPC-P25 & $C_{1}=1000$ & -29.0 \\
& $C_{2}=100$ & -35.1 \\
& $C_{3}=10$ & -39.4 \\
\end{tabular}

DPPC coating increases the size of NPs by $30 \%(21 \mathrm{~nm}+10 \mathrm{~nm}$ DPPC bilayer coating) and consequently, the density of the DPPC-P25 NPs must be smaller. For example, if the density of a $\mathrm{P} 25 \mathrm{NP}$ is $\rho_{\mathrm{P} 25}=3.8 \mathrm{~g} \mathrm{~cm}^{-3}$ and the density of the DPPC coating $\rho_{\text {DPPC }}=1.03 \mathrm{~g} \mathrm{~cm}^{-3}$, the density of a DPPC-P25 NP will be reduced to half the density value of P25 NP $\left(\rho_{\text {DPPC-P25 }} \cong 1.9\right)$. This reduction of the DPPC-P25 NP density implies that the measured BET specific surface area is actually twice the value shown in Table 3.

The second factor which could contribute to the large decrease in specific surface area of DPPC-P25 NPs is the potential of the coating to cover/block the pores present on the surface of the P25 NPs. BET surface area depends on the amount of $\mathrm{N}_{2}$ gas adsorbed in the pores of the NPs surface. P25 NPs have pore volume of $0.25 \mathrm{~cm}^{3} \mathrm{~g}^{-1}$ and pore size of $17.5 \mathrm{~nm}$ (ref. 51) and it is mesoporous according to IUPAC classification (size of pores 2-50 nm). By covering the pores and the adsorption sites on the P25 NPs, the DPPC coating can reduce the roughness and therefore, the specific surface area of NPs.

The hydrophilicity (water wettability) and the surface charge of NPs are important factors which influence their dispersibility and colloidal stability in water. To further investigate the effect of the DPPC coating on the P25 NPs, dispersions of NPs in DI water were prepared in a range of three concentrations relevant to the toxicology studies. The surface charge of NPs and the size distribution of the dispersions were measured with the Zetasizer and the CPS centrifuge.

The zeta potential results listed in Table 3 indicate that the DPPC-P25 NPs have the largest measured negative surface charge values in all three concentrations. The $\mathrm{pH}$ of the dispersions was approximately 7 and all zeta potential values are at or above the threshold value for stable dispersions $(-30 \mathrm{mV})$.

Fig. 5a and c show the particle size distribution measured with the CPS centrifuge for the pristine and DPPC coated P25 dispersions in three concentrations. The CPS centrifuge results shown in Fig. 5a indicate that P25 dispersions in DI water were broadly polydispersed (PSD spreading between 0.08 and $5 \mu \mathrm{m}$ ). The results measured with Malvern Zetasizer (Fig. 5b) were not qualitatively acceptable because of the polydispersity of the samples.

The DPPC-P25 NP dispersions measured with CPS centrifuge (Fig. 5c) and Malvern Zetasizer (Fig. 5d) show low average sizes and narrow distributions for all three concentrations 

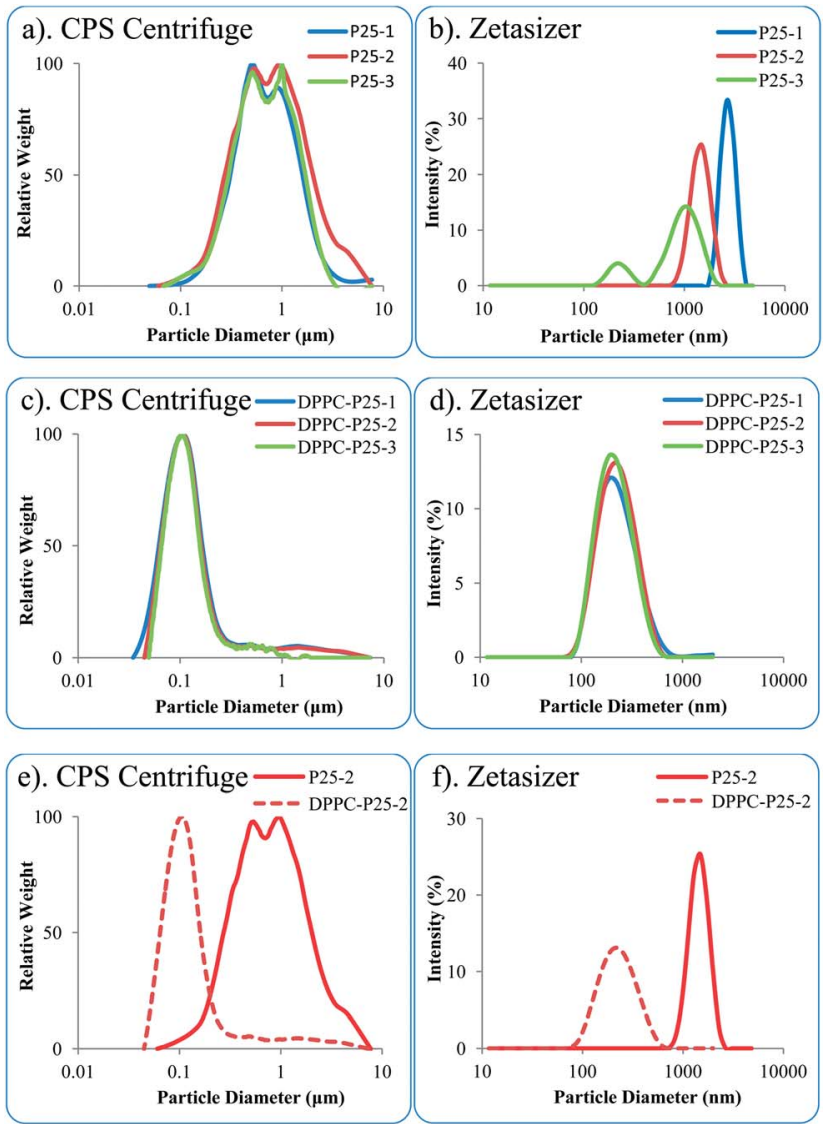

Fig. 5 Particle size distribution of NP dispersions in DI water (a) P25 $C_{1}$ $=1, C_{2}=0.1, C_{3}=0.01 \mathrm{mg} \mathrm{m}^{-1}$ dispersions measured with CPS centrifuge, (b) P25 $C_{1}=1, C_{2}=0.1, C_{3}=0.01 \mathrm{mg} \mathrm{ml}^{-1}$ dispersions measured with Malvern Zetasizer, (c) DPPC-P25 $C_{1}=1, C_{2}=0.1, C_{3}=$ $0.01 \mathrm{mg} \mathrm{ml}^{-1}$ dispersions measured with CPS Centrifuge, (d) DPPCP25 $C_{1}=1, C_{2}=0.1, C_{3}=0.01 \mathrm{mg} \mathrm{ml}^{-1}$ dispersions measured with Malvern Zetasizer, (e) P25 and DPPC-P25 $C_{2}=0.1 \mathrm{mg} \mathrm{ml}^{-1}$ dispersions measured with CPS centrifuge and (f) P25 and DPPC-P25 $C_{2}=$ $0.1 \mathrm{mg} \mathrm{ml}^{-1}$ dispersions measured with Malvern Zetasizer.

tested. In this case the Malvern Zetasizer results were qualitatively acceptable and the size distribution was more similar to that measured by the CPS centrifuge. Both instruments measure the dispersions hydrodynamic diameter which is controlled by NPs agglomeration (function of zeta potential and ionic strength of dispersion media) in the aqueous system but also a strong function of primary particle size. Published research studies ${ }^{38}$ which also employed the Malvern Zetasizer to investigate titania NP dispersions in DI water showed that even when the dispersions were stable (the zeta potential was $30 \mathrm{mV}$ ), the measured average hydrodynamic diameter was much larger than the primary particle size (the measured average hydrodynamic diameter of NPs with primary size of $6 \mathrm{~nm}$ was $67 \mathrm{~nm}$ ).

Fig. 5e and $\mathrm{f}$ compare PSDs measured with the CPS Centrifuge and Malvern Zetasizer of $0.1 \mathrm{mg} \mathrm{ml}^{-1}\left(C_{2}\right)$ P25 and DPPCP25 dispersions in DI water. The DPPC-P25 dispersion has a small average particle size and a narrow distribution while the P25 dispersion has large average particle size (forms large aggregates) and is broadly polydispersed. The Zetasizer distribution results for the P25 dispersions did not meet the quality criteria because the polydispersity index was too high (the size distribution for $C_{2}$ dispersion was plotted in Fig. $5 \mathrm{f}$ for comparison only).

The particle size distribution and zeta potential results indicate that the DPPC coating increases the surface charge and reduces both, the average hydrodynamic diameter and polydispersity of the P25 NP dispersions, therefore generating a more stable NP dispersion. These results correlate very well with the heat of wetting values which also show a large increase in the wettability of P25 NPs when coated with a complete bilayer of DPPC.

\subsection{Effect of DPPC coating on cytotoxicity of NPs}

Fig. 6a and b show the 24 hours A549 cell viability versus nanoparticle concentration and the SDs of the pristine and DPPC coated P25 using the LDH and MTT assays.

The results show the effect of the DPPC coating on the NP cytotoxicity. One way Anova reveals that in the LDH and MTT assays, P25 NPs are significant different from DPPC-P25.

The toxicity of DPPC-P25 NPs is reduced in the entire concentration range tested and reached $25 \%$ (75\% viability) at high NP concentrations (above $100 \mu \mathrm{g} \mathrm{cm}^{-1}$ ). For the DPPC-P25 NPs there is a slight downshift in the viability curve for the top two concentrations but these doses are very high as the highest standard dose is typically $333 \mu \mathrm{g} \mathrm{cm}^{-2}$.

Several studies employed DPPC concentration which enabled the complete coverage of NPs and demonstrated the protective effects of lung surfactant coating on toxic particles (silica etc.). Wallace and et al. ${ }^{15}$ showed that the DPPC treated quartz and kaolin particles incubated for 1 hour with erythrocytes suppressed the hemolytic strength of particles to background levels. Studies on pulmonary alveolar macrophages showed the DPPC fully suppressed silica NPs prompt cytotoxic, apoptotic, necrotic and genotoxic activity for 3 days..$^{15,16,33,52}$ The interaction between DPPC and different types of silica dust has been suggested to modulate the toxicity of the particles through the molecular orientation of the adsorbed DPPC molecules. This may account for differences in pulmonary disease abnormalities. ${ }^{21}$ The artificial surfactant Survanta (FDA approved pulmonary surfactant extracted from minced cow lung with additional DPPC, palmitic acid and tripalmitin) protected alveolar macrophages in vitro and reduced lung injury in vivo without affecting neutrophil influx. ${ }^{53}$ In the special case of crysotile asbestos, the prophylactic effect of the DPPC coating was
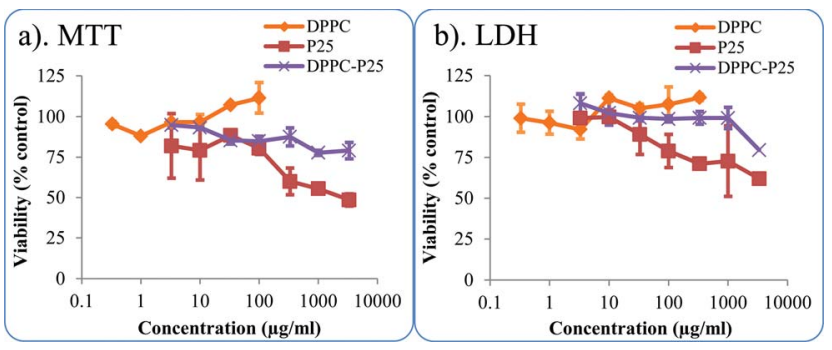

Fig. 6 A549 cells viability exposed to pristine and DPPC coated P25 NPs (a) MTT assay and (b) LDH assay. 
found ineffective at inhibiting its toxicity ${ }^{33}$ despite the reported ability to adsorb on its surface. ${ }^{54}$ This finding reveals that the toxicity of crysotile asbestos may thus depend on parameters other than the surface properties such as the shape of particles.

The protective effect against toxic particle induced lungdamage is only temporary as research showed that subsequent removal of the prophylactic DPPC coating through secondary lysosomal or extracellular PLA-2 phospholipase enzymatic digestion restored the toxicity in 3-7 days. ${ }^{15,17-20,33,55}$ These differences were ascribed to variations in the rate of digestion by lysosomal enzymes.

It was emphasized in toxicology studies that critical toxic events in mineral dust-induced fibrosis occur in cells in the alveolar interstitium rather than in the macrophages of the alveolar surface hypophase $\mathrm{s}^{56}$ and investigations should also be carried out on pulmonary alveolar interstitial cells. ${ }^{19}$ Sager $^{1}$ reported that data from their laboratory indicate that improved dispersion of SWCNTs resulted in significant enhanced potency of aspirated material to induce interstitial fibrosis in mice.

Apart from the damage of lysosomes by the phagocytized cytotoxic particles another important reaction of the macrophages with particles is the production and release of reactive oxygen species (ROS). Hertog ${ }^{5,23}$ and Foucaud ${ }^{24}$ employed DPPC concentrations which according to our calculations enabled complete monolayer coverage of their studied hydrophobic NPs, SWCNT and CB respectively and reported an improved particle dispersion which was considered the reason for the increased ROS production in A549 cells and DCFT oxidation in MonoMac6 human cells.

Numerous reported studies showed that dispersion of NPs (especially CNTs) is critical to their effective distribution in the lung. In line with these studies, our results revealed that P25 NPs coated with a complete DPPC bilayer become more hydrophilic and generate stable dispersions with an average hydrodynamic diameter of 110-190 nm. Published TEM images of aluminum particles inside an endosome of A549 cell from in vivo studies also showed NP aggregates with similar sizes (100$200 \mathrm{~nm}) .{ }^{27}$ This emphasizes the importance of conditioning the NP surface in toxicology studies to mimic pulmonary exposure more closely. Employing DPPC in concentrations which enable a complete coverage of NPs is an important step in achieving this aim. The establishment of standard dispersion media and protocols will enable researchers to achieve a degree of concordance and hence, could greatly benefit nanotoxicology research.

\section{Conclusions}

The enthalpy of wetting includes two NP parameters highly relevant to toxicology, wettability and surface area and along with surface charge can be used to predict their dispersibility and stability in aqueous fluids and consequently, their interaction with biological systems.

On titania P25 NPs, the adsorption of a complete DPPC bilayer has had the following critical effects:

(1) Increased hydrophilicity of NPs and aided their dispersion in aqueous media.
(2) Reduces the specific surface area of dry NPs.

(3) Reduced the toxicity of NPs on A549 cells in 24 hour tests.

To mimic the in vivo pulmonary conditions more closely and enable the replication of the surfactant in vivo action, nanotoxicology studies should employ the most abundant surface active component of the lung surfactant, DPPC in a DPPC/NPS wt ratio which enables complete coverage of NPs. Following this testing strategy will hopefully lead to reaching consensus in cytotoxicity results.

The investigation of DPPC adsorption on P25 titania NPs has provided important information on their wettability, charge and dispersibility in biological fluids with consequences on the lung cells uptake and toxicity. This study represents a first step in establishing a protocol for nanotoxicity testing and we recommend similar tests to be carried out on hydrophobic NPS along with investigating the effect of the relevant SPs on the DPPC adsorption and particle toxicity.

\section{References}

1 T. M. Sager, D. W. Porter, V. A. Robinson, W. G. Lindsley, D. E. Schwegler-Berry and V. Castranova, Improved method to disperse nanoparticles for in vitro and in vivo investigation of toxicity, Nanotoxicology, 2007, 1(2), 118-129.

2 A. Shvedova, T. Sager, A. Murray, E. Kisin, D. Poster, S. Leonard, D. Schwegler-Berry, V. Robinson and V. Castranova, Critical issues in the evaluation of possible adverse pulmonary effects resulting from airborne nanoparticles, in Nanotoxicology: Characterization, Dosing and Health Effects, ed. N. A. Monteiro-Riviere and C. L. Tran, Informa Healthcare, New York, London, 2007, pp. 225-236.

3 P. Wick, P. Manser, L. K. Limbach, U. Dettlaff-Weglikowska, F. Krumeich, S. Roth, W. J. Stark and A. Bruinink, The degree and kind of agglomeration affect carbon nanotubes toxicity, Toxicol. Lett., 2007, 168(2), 121-131.

4 D. Porter, K. Sriram, M. Wolfarth, A. Jefferson, D. SchweglerBerry, M. Andrew and V. Castranova, A biocompatible medium for nanoparticle dispersion, Nanotoxicology, 2008, 2(3), 144-154.

5 E. Herzog, H. J. Byrne, A. Casey, M. Davoren, A. G. Lenz, K. L. Maier, A. Duschl and G. J. Oostingh, SWCNT suppress inflammatory mediator responses in human lung epithelium in vitro, Toxicol. Appl. Pharmacol., 2009a, 234, 378-390.

6 W. E. Wallace, M. J. Keane, D. K. Murray, W. P. Chisholm, A. D. Maynard and T. Ong, Phospholipid lung surfactant and nanoparticle surface toxicity: Lessons from diesel soots and silicate dusts, J. Nanopart. Res., 2007b, 9, 23-38.

7 M. C. Bufford, R. F. Hamilton and A. Holian, A comparison of dispersing media for various engineering carbon nanoparticles, Part. Fibre Toxicol., 2007, 4(6), 1-9.

8 J. S. Patton, Mechanisms of macromolecule adsorption by the lungs, Adv. Drug Delivery Rev., 1996, 19, 3-36.

9 R. Veldhuizen, K. Nag, S. Orgeig and F. Possmayer, The role of lipids in pulmonary surfactant, Biochim. Biophys. Acta, 1998, 1408, 90-108. 
10 S. Schurch, P. Gehr, V. I. Hof, M. Geiser and F. Green, Surfactant displaces particles toward the epithelium in airways and alveoli, Respir. Physiol., 1990, 80, 17-32.

11 S. Schurch, H. Bachofen, J. Groeke and F. Green, Surface properties of rat pulmonary surfactant studied with the captive bubble method: adsorption, hysteresis, stability, Biochim. Biophys. Acta, 1992, 1103, 127-136.

12 W. R. Schief, M. Antia, B. M. Discher, S. B. Hall and V. Vogel, Liquid crystalline collapse of pulmonary surfactant monolayers, Biophys. J., 2003, 84, 3792-3806.

13 F. Possmayer, Pulmonary Perspective. A Proposed Nomenclature for Pulmonary Surfactant-associated Proteins, Am. Rev. Respir. Dis., 1988, 138, 990-998.

14 W. E. Wallace, M. J. Keane, C. A. Hill, J. Xu and T. M. Ong, Mutagenicity of diesel exhaust particles and oil shale particles dispersed in lecithin surfactant, J. Toxicol. Environ. Health, 1987, 21, 163-171.

15 W. E. Wallace, M. J. Keane, V. Vallyathan, P. Hathaway, E. D. Regad, V. Castranova and F. H. Y. Green, Suppression of Inhaled particles cytotoxicity by pulmonary surfactants and re-toxification by phospholipase: distinguishing properties of quartz and kaolin, Ann. Occup. Hyg., 1988, 32, 291-298.

16 W. E. Wallace, M. J. Keane, P. S. Mike, C. A. Hill, V. Vallyathan and E. D. Regad, Contrasting respirable quartz and kaolin retention of lecithin surfactant and expression of membranolitic activity following phopholipase A2 digestion, J. Toxicol. Environ. Health, 1992, 37, 391-409.

17 C. A. Hill, W. E. Wallace, M. J. Keane and P. S. Mike, The enzymatic removal of a surfactant coating from quartz and kaolin by P388D1 cells, Cell Biol. Toxicol., 1995, 11, 119-128.

18 X. Liu, M. J. Keane, J. C. Harrison, E. V. Cilento, T. Ong and W. E. Wallace, Phospholipid surfactant adsorption by respirable quartz and in vitro expression of cytotoxicity and DNA damage, Toxicol. Lett., 1998, 96(97), 77-84.

19 N. Gao, M. J. Keane, T. Ong and W. E. Wallace, Effects of Simulated Pulmonary surfactant on the cytotoxicity and DNA-damaging activity of the respirable quartz and Kaolin, J. Toxicol. Environ. Health, Part A, 2000, 60, 153-167.

20 N. Gao, M. J. Keane, T. Ong, J. Ye, W. E. Miller and W. E. Wallace, Effects of Phospholipid Surfactant on Apoptosis Induction by Respirable Quartz and Kaolin in NR8383 rat pulmonary macrophages, Toxicol. Appl. Pharmacol., 2001, 175, 217-225.

21 D. K. Murray, J. C. Harrison and W. E. Wallace, A 13C CP/ MAS and 31P NMR study of the interactions of dipalmitoylphosphatidylcholine with respirable silica and kaolin, J. Colloid Interface Sci., 2005, 288, 166-170.

22 W. E. Wallace, M. J. Keane, M. Guatam, X. C. Shi, D. Murray and $\mathrm{T}$. Ong, Dispersion of nanoparticles in pulmonary surfactants for in vitro toxicity studies: lessons from ultrafine diesel exhaust particles and fine mineral dusts, in Nanotoxicology: Characterization, Dosing and Health Effects, ed. N. A. Monteiro-Riviere and C.L. Tran, Informa Healthcare, New York, London, 2007, pp. 153-172.

23 E. Herzog, H. J. Byrne, M. Davoren, A. Casey, A. Duschl and G. J. Oostingh, Dispersion medium modulates oxidative stress response of human lung epithelial cells upon exposure to carbon nanomaterial samples, Toxicol. Appl. Pharmacol., 2009b, 236, 276-281.

24 L. Foucaud, M. R. Wilson, D. M. Brown and V. Stone, Measurement of reactive species production by nanoparticles prepared in biologically relevant media, Toxicol. Lett., 2007, 174, 1-9.

25 M. Ratoi, H. A. Spikes and H. L. Rieffe, Optimizing film formation by oil-in-water emulsions, Tribol. Trans., 1997, 40(4), 569-578.

26 B. P. Binks, L. Isa and A. T. Tyowua, Direct measurement of contact angles of silica particles in relation to double inversion of pickering emulsions, Langmuir, 2013, 29, 4923-4927.

27 K. W. Powers, S. C. Brown, V. B. Krishna, S. C. Wasdo, B. M. Moudgil and S. M. Roberts, Research strategies for safety evaluation of nanomaterils. Part VI. Characterization of Nanoscale Particles for Toxicological Evaluation, Toxicol. Sci., 2006, 90(2), 296-303.

28 G. D. Parfitt and D. G. Wharton, The dispersion of rutile powder in aqueous surfactant solutions, J. Colloid Interface Sci., 1972, 38(2), 431-439.

29 J. N. Israelachvili and G. E. Adams, Measurement of forces between 2 mica surfaces in aqueous electrolyte solutions in range 0-100 nm, J. Chem. Soc., Faraday Trans., 1978, 74(1), 975-1001.

30 F. Tiberg, Physical characterization of non-ionic surfactant layers adsorbed at hydrophilic and hydrophobic solid surfaces by time-revolved ellipsometry, J. Chem. Soc., Faraday Trans., 1996, 92, 531-538.

31 V. Oliynyk, U. Kaaze and T. Heimburg, Defect formation of lytic peptides in lipid membranes and their influence on the thermodynamic properties of the pore environment, Biochim. Biophys. Acta, 2007, 1768, 236-245.

32 M. Kendall, T. D. Tetley, E. Wigzell, B. Hutton, M. Nieuwenhuijsen and P. Luckham, Lung lining liquid modifies PM2.5 in favor of particle aggregation: a protective mechanism, Am. J. Physiol. Lung Cell Mol. Physiol., 2002, 282, L109-L114.

33 J. Schimmelpfeng, E. Drosselmeyer, V. Hofheinz and A. Seidel, Influence of surfactant components and exposure geometry on the effects of quartz and asbestos on alveolar macrophages, Environ. Health Perspect., 1992, 97, 225-231.

34 T. D. Blake, Wetting, in Surfactants, ed. T. F. Tadros, Academic Press, Harcort Brace Jovanovich Publ., 1984, ch.10, pp. 221-276.

35 T. W. Healy and D. W. Fuerstenau, The oxide-water interfaceinterrelation of the zero point of charge and the heat of immersion, J. Colloid Sci., 1965, 20(4), 376-386.

36 J. Geys, B. Nemery and P. H. Hoet, Assay conditions can influence the outcome of cytotoxicity tests of nanomaterials: better assay characterization is needed to compare studies, Toxicol. in Vitro, 2010, 24(2), 620-629.

37 S. Lanone, F. Rogerieux, J. Geys, A. Dupont, E. MaillotMarechal, J. Boczkowski, G. Lacroix and P. Hoet, Comparative toxicity of 24 manufactured nanoparticles in 
human alveolar epithelial and macrophage cell lines, Part. Fibre Toxicol., 2009, 6(14), 1-12.

38 K. Suttiponparnit, J. Jiang, M. Sahu, S. Suvachittanont, T. Charinpanitkul and P. Biswas, Role of surface area, primary particle size and crystal phase on titanium dioxide nanoparticle dispersion properties, Nanoscale Res. Lett., 2011, 6(27), 1-8.

39 S. Ahmed and S. L. Wunder, Effect of High surface Curvature on the Main Phase Transition of Supported Phospholipid Bilayers on $\mathrm{SiO}_{2}$ Nanoparticles, Langmuir, 2009, 5, 36823691.

40 L. Opilik, T. Bauer, T. Schmid, J. Stadler and R. Zenobi, Nanoscale Chemical Imaging of Segregated Lipid Domains using Tip-Enhanced Raman Spectroscopy, Phys. Chem. Chem. Phys., 2011, 13, 9978-9981.

41 E. N. Lewis, R. Bittman and I. W. Levin, Methyl group substitution at $\mathrm{C}(1), \mathrm{C}(2)$ or $\mathrm{C}(3)$ of the glycerol backbone of a diether phosphocholine: a comparative study of bilayer chain disorder in the gel and liquid-crystalline phases, Biochim. Biophys. Acta, 1986, 861(1), 44-52.

42 S. Ahmed, S. L. Wunder and Z. S. Nickolov, Raman Spectroscopy of Supported Lipid Bilayer Nanoparticles, Spectroscopy, 2011, Special Issues, June, http:// www.spectroscopyonline.com/spectroscopy/article, retrieved on 30th October 2013.

43 J. M. Douillard and J. Zajac, Contact Angle Determination from Heat of Immersion and Heat of Wetting, in Encyclopedia of Surface and Colloidal Science, ed. P. Somasundaran, Marcel Dekker, New York, Update Supplement, 2004, pp. 153-163.

44 G. Buckton, Characterization of small changes in the physical properties of powders of significance for dry powder inhaler formulations, Adv. Drug Delivery Rev., 1997, 26, 17-27.

45 Y. W. Yang, G. Zografi and E. E. Miller, Capillary flow phenomena and wettability in porous media II. Dynamic flow studies, J. Colloid Interface Sci., 1988, 122, 35-46.

46 R. M. Shanker, P. J. Baltusis and R. M. Hruska, Development of a new technique for the assessment of wettability of powders, Pharm. Res., 1995, 11, s-243.
47 T. N. Hunter, G. J. Jameson and E. J. Wanless, Determination of contact angles of nanosized silica particles by multi-angle single-wavelength ellipsometry, Aust. J. Chem., 2007, 60, 615655.

48 L. Isa, F. Lucas, R. Wepf and E. Reimhult, Measuring singlenanoparticle wetting properties by freeze-fracture shadowcasting cryo-scanning electron microscopy, Nat. Commun., 2011, 2(438), 1-9.

49 D. W. Fuerstenau and S. Raghavan, Some aspects of flotation thermodynamics, in Froth Flotation, A Century of Innovation, ed. M. C Fuerstenau, G. Jameson and R.-H. Yoon, Society of Mining, Metallurgy and Exploration (SME), Colorado, USA, 2007, pp. 95-132.

50 M. Jurak and E. Chibowaki, Wettability and topography of phospholipid DPPC multilayers deposited by spin-coating on glass, silicon and mica slides, Langmuir, 2007, 23, 10156-10163.

51 K. J. A. Raj and B. Viswanathan, Effect of surface area, pore volume and particle size of P25 titania on the phase transformation of anatase to rutile, Indian J. Chem., Sect. A: Inorg., Bio-inorg., Phys., Theor. Anal. Chem., 2009, 48, 13781382.

52 S. Patzold, A. Schmidt and A. Seidel, Loss of cathepsin B activity in alveolar macrophages after in vitro quartz phagocytosis, J. Toxicol. Environ. Health, 1993, 40, 547-554.

53 J. M. Antonini and M. J. Reasor, Effect of short-term exogenous pulmonary surfactant treatment of acute lung damage associated with the intratracheal instillation of silica, J. Toxicol. Environ. Health, 1994a, 43, 85-101.

$54 \mathrm{~J}$. Bignon and M. C. Jaurand, Biological in vitro and in vivo responses of chrysotile versus amphiboles, Environ. Health Perspect., 1983, 51, 73-80.

55 J. M. Antonini, C. M. McCloud and M. J. Reasor, Acute silica toxicity: attenuation by amiodarone-induced pulmonary phospholipidosis, Environ. Health Perspect., 1994b, 102, 372-378.

56 I. Y. R. Adamson, H. L. Letourneau and D. H. Bowden, Enhanced macrophage-fibroblast interactions in the pulmonary interstititum increase fibrosis after silica injection to monocyte-depleted mice, Am. J. Physiol., 1989, 134, 411-417. 